\title{
An iterative approach to the thermal Newtonian blown film model
}

\author{
$\begin{array}{lll}\text { J. C. Bennett } & \text { J. J. Shepherd } & \text { W. F. Blyth }\end{array}$
}

(Received 31 August 2006; revised 19 December 2007)

\begin{abstract}
Film blowing, by which thin polymer film is manufactured, is an industrial process with products used in a wide range of applications. Modelling of this process requires solving the highly nonlinear differential equations necessary to describe the complex chemical and physical interactions involved. Earlier work applied a mixture of analytic and numerical techniques to this modelling when the polymer involved was a Newtonian fluid operating under isothermal conditions. Subsequent calculations extended this analysis to the non-isothermal Newtonian film. However, the numerical procedures used were found to be computationally expensive. We apply a more robust finite element approach to these computations, that avoids the difficulties of the earlier methods, and is an improvement on these previous efforts.
\end{abstract}

See http://anziamj.austms.org.au/ojs/index.php/ANZIAMJ/article/view/120 for this article, (c) Austral. Mathematical Soc. 2008. Published January 5, 2008. ISSN 1446-8735 


\section{Contents}

1 Introduction

C838

2 Non-dimensional governing equations

C840

3 Analytic approximation

C841

4 Linearization and the iterative scheme

C842

5 Results and discussion

C845

References

C849

\section{Introduction}

The basic elements of the film blowing process are very simple. Molten polymer is extruded through an annular die, with an applied internal air pressure causing the film to expand in radius as it is drawn upwards. The resulting polymer 'bubble' is cooled by air rings located above the die, causing the film to solidify. Subsequently, the film is drawn off onto rollers as a double layered sheet. The point of film solidification of the polymer is usually referred to as the freezeline.

Film blowing has been widely investigated, both mathematically and experimentally. Pearson and Petrie $[6,7,8]$ provide a basis for most of the models through their analysis based on thin shell theory. Muke et al. [5] briefly reviewed the literature. The model considered here is a simplified one, in that the polymer film blown is assumed to be an incompressible isothermal Newtonian fluid, with gravity effects omitted. This simplified model has the benefit of being relatively accessible to analysis, while retaining much of the solution structure seen in more sophisticated models. 
A key feature in the analysis arising in modelling film blowing has been the need to numerically solve the nonlinear, two point, boundary value problem defining the film bubble radius. Typically such solutions are found via a shooting method; however, instabilities have been reported by Luo and Tanner [4]. Investigation [1, 2, 9, 10, 11] showed that, under appropriate conditions, the film bubble radius profile exhibited an internal 'layer' region, possibly leading to numerical instabilities. A mixture of analytic methods exploiting this structure led to the construction of an explicit expression for the bubble radius that approximated known numerical results with acceptable accuracy.

Bennett and Shepherd [3] subsequently investigated the case of a Newtonian film under non-isothermal conditions; that is, a temperature profile existed along the film profile. In this case, the bubble radius and temperature differential equations are coupled; but were de-coupled by means of a cross-linked iterative scheme. Thus, at each stage of iteration, the nonlinear boundary value problem for the radius profile was to be solved. The isothermal analytic expression constructed by Shepherd and Bennett [9] was then used as a starting approximation for the radius profile, and a more accurate solution was then sought by linearization of the full nonlinear radius problem about this approximation and numerical solution by a further (sub)iteration process. In this, it was reasoned [3] that most of the layer structure lay in this analytic expression, so that numerical construction of a (small) correction was much less likely to encounter instabilities. This correction was found using Maple's internal boundary value problem solver, but this was found to be computationally expensive at each iterate stage.

This article improves on the results by Bennett and Shepherd [3], by applying a different boundary value problem solving technique. For this, a Galerkin finite element technique was employed when obtaining the correction described above. An acceptable solution was obtained with computation time being radically reduced. 


\section{Non-dimensional governing equations}

The model assumptions and simplifications are the same as those of Bennett and Shepherd [3], with the problem geometry as illustrated by Shepherd and Bennett [9]. Under suitable assumptions, the non-dimensional state variables for the process are the film radius, velocity and temperature, $r(z)$, $u(z)$ and $s(z)$, respectively, with $z$ the distance from the die along the bubble axis. The non-dimensional governing equations linking these are then [3]

$$
\begin{aligned}
2 C^{2} r^{2}\left[f_{0}+B\left(r^{2}-1\right)\right] r^{\prime \prime}-6 C \eta r^{\prime} & \\
-r\left[f_{0}-B\left(3 r^{2}+1\right)\right]\left[1+C^{2}\left(r^{\prime}\right)^{2}\right] & =0, \\
2 C\left(2 r u^{\prime}+u r^{\prime}\right)-\frac{r u}{\eta}\left[f_{0}+B\left(r^{2}-1\right)\right]\left[1+C^{2}\left(r^{\prime}\right)^{2}\right] & =0, \\
s^{\prime}+H r \sqrt{1+C^{2}\left(r^{\prime}\right)^{2}}\left(s-s_{a}\right)+J r \sqrt{1+C^{2}\left(r^{\prime}\right)^{2}}\left(s^{4}-s_{a}^{4}\right) & =0,
\end{aligned}
$$

subject to the conditions

$$
\begin{aligned}
& r(0)=1, \quad r^{\prime}(1)=0, \\
& u(0)=1, \quad s(0)=1 .
\end{aligned}
$$

Here, our dimensionless parameters are $B, C, f_{0}, H, J, w$ and $s_{a}$. The parameter $B$ arises from the applied internal air pressure difference, so is to be regarded as a pressure parameter. Parameter $C$ is the geometric ratio of the bubble radius at the die to the axial distance to the freezeline. Since the distance to the freezeline is much greater than the exit radius, $C$ is regarded as small. Parameter $f_{0}$ is the scaled pulling force on the film at the die exit. The parameters $H, J, w$ and $s_{a}$ relate to the thermal variation along the film. Parameter $H$ is a scaled heat transfer coefficient, $J$ is a scaled emissivity, $w$ is a scaled activation energy and $s_{a}$ is a scaled ambient temperature. The thermal variation is introduced into Equations (1) and (2) through the Arrhenius factor

$$
\eta(z)=\exp \left[w\left(\frac{1}{s}-1\right)\right] .
$$


The differential equations (1) and (3) are coupled, as opposed to those of the isothermal case [9] (which is recovered by substituting $s(z) \equiv 1$ into $\eta(z)$ ). As was shown by Bennett and Shepherd [3], these equations are decoupled through the iteration scheme employed.

\section{Analytic approximation}

Our linearization procedure is built around having an approximation with a similar layer structure to that demonstrated in numerical solutions. As noted in Section 1, this approximation was constructed for the isothermal case using a mixture of analytic methods and assumptions. For this case, a split interval solution was obtained which displayed acceptable structure. This was then used as a basis for an iteration scheme to construct a solution for the thermal Newtonian case [3]. Here, we again use our isothermal split interval solution to iterate towards our numerical solution; but here we employ a much more efficient numerical iteration process. Our closed form approximate solution for the radial profile is

$$
r_{c}(z)=r_{1}(z)(H(z)-H(z-a))+r_{2}(z)(H(z-a)-H(z-1)) .
$$

Here, $H(z)$ is the Heaviside function, and

$$
\begin{aligned}
r_{1}(z, C)= & \phi(z, C)+[\phi(a, C)-\lambda] \operatorname{erf}\left[\frac{\sqrt{2 M}(a-z)}{2 C}\right] \\
& +\lambda-\phi(a, C),
\end{aligned}
$$

with

$$
\phi(z, C)=\left\{\left(1-\frac{3 B}{f_{0}-B}\right) \exp \left[\left(\frac{f_{0}-B}{3 C}\right) z\right]+\frac{3 B}{f_{0}-B}\right\}^{-1 / 2} ;
$$

and

$$
r_{2}(z, C)=\lambda+\frac{1}{18} \rho_{B U}^{3} \sigma \gamma\left[e^{\nu(z, C)}-e^{\nu(a, C)}\right]-\frac{\rho_{B U}}{6 C} \gamma(z-a),
$$


where

$$
\begin{aligned}
& \sigma=f_{0}+B\left(\rho_{B U}^{2}-1\right) \\
& \gamma=f_{0}-B\left(3 \rho_{B U}^{2}+1\right)
\end{aligned}
$$

while

$$
\nu(z, C)=\frac{3(z-1)}{C \sigma \rho_{B U}^{2}} .
$$

The $a$ and $\lambda$ occurring in the analytic approximation above arise from imposing continuity at the point of change of region; that is,

$$
r_{1}(a, C)=r_{2}(a, C)=\lambda
$$

and are found by requiring smoothness of join at the change point $z=a$, together with the requirement that the approximation attain the value $\rho_{B U}$ at $z=1$, that is,

$$
r_{1}^{\prime}(a, C)=r_{2}^{\prime}(a, C), \quad r_{2}(1, C)=\rho_{B U},
$$

where $\rho_{B U}$ is the 'blow-up' ratio, or scaled film radius at the freezeline.

The benefits of such an explicit approximate representation of the bubble radius profile as a start for a numerical iterative scheme are outlined in Section 1. Note that the coupled Equations (1) and (3) may in principle be solved as a coupled boundary value problem, but in practice a solution is frequently unobtainable, often as a result of the instabilities described above arising from the solution's layer structure.

\section{Linearization and the iterative scheme}

The linearization scheme employed here is the same as that by Bennett and Shepherd [3]. In short, we assume an initial estimate to the solution of 
Equation (1) and expand around this; that is, we put

$$
r(z)=R(z)+v(z),
$$

where $R(z)$ is this initial approximation $\left(R(z)=r_{c}(z)\right.$ in our case) and $v(z)$ is a correction term. We substitute this into Equation (1), expand whilst holding $\eta(z)$ fixed (recall that $\eta$ depends on the temperature $s$ ) and collect the linear terms involving $v(z)$ on the left hand side and the residual and nonlinear (quadratic and higher) terms in $v(z)$ together on the right hand side. This gives a rearranged form of Equation (1) as

$$
a(R) v^{\prime \prime}+b(R, s) v^{\prime}+c(R) v=N(R, s)+Q\left(R, v, v^{\prime}, v^{\prime \prime}\right),
$$

where $v(z)$ satisfies the boundary conditions

$$
v(0)=0, \quad v^{\prime}(1)=0 .
$$

We now set up our basic iteration scheme as follows. From Equation (17) we form the associated equation

$$
a(R) v_{n}^{\prime \prime}+b(R, s) v_{n}^{\prime}+c(R) v_{n}=N(R, s)+Q\left(R, v_{n-1}, v_{n-1}^{\prime}, v_{n-1}^{\prime \prime}\right) .
$$

where $n=1,2, \ldots$ For given $v_{n-1}, s$ and $R$, Equation (19) is a linear equation for $v_{n}$, which is solved (numerically), subject to the boundary conditions (18). Thus, given an initial iterate $v_{0}(z)$, a sequence $v_{0}, v_{1}, v_{2}, \ldots$ of approximations is constructed, which, hopefully, converges to the solution of the problem (17) and (18). Any of these approximations may be applied to (16) to yield successive approximations to the bubble radius $r(z)$; and this may be used in (3), to approximate the temperature profile $s(z)$.

The success of the process described above clearly depends on our choice of $v_{0}(z)$ (we choose $v_{0}(z)=0$ here), and the initial approximations for $s(z)$ and $R(z)$. As noted above, we initially choose $R(z)=r_{c}(z)$, and an initial approximation for the temperature variation, $s(z)$, is found by solving Equation (3) subject to $s(0)=1$ with $r(z)=r_{c}(z)$. 
Convergence of the process is enhanced by stopping the iteration at iterate $n$ and updating the $R(z)$ in (19) via an application of (16); that is,

$$
R_{\text {new }}(z)=R_{\text {old }}(z)+v_{n}(z),
$$

while an updated $s(z)$ is obtained by solving (3) subject to $s(0)=1$ with $r(z)=R_{\text {new }}(z)$. Iteration may then be resumed with $v_{0}=0$.

This process is somewhat ad hoc, in that there is no clear indication of how many iterates to perform before $R$ and $s$ need to be updated. We found that three iterations before updating were sufficient. Other procedures might be employed at the possible cost of increased computation time. For the purposes of reporting results, we will refer to each update of $R$ and $s$ as a changing of 'stage'. Further, we use a comparison of successive iterates within a particular stage as a measure of convergence within that stage.

The linear differential equation (19) was solved using a Galerkin finite element method, with piecewise linear shape functions. The method is easily adaptable to any shape function but the hat functions used here proved sufficient. To achieve good accuracy only required 200 nodes. Data used (as in the substitution of $R$ and $s$ into (19) was first interpolated using quintic splines. Odd degree splines were chosen because the knots coincide with the position of the nodes whereas even splines choose a fictitious nodal point as the midpoint of the nodes and the knot is defined there. Fourth order continuity was enforced at each nodal point. Fifth order splines were used because of the second order derivative terms in $v(z)$ that appear in $Q\left(R, v, v^{\prime}, v^{\prime \prime}\right)$. If a third order spline had been used, continuity at each of the nodes would be enforced to second order but smoothness only to first order. Hence fifth order was chosen for smoothness of the system. 
TABLE 1: Table of maximum values of the difference of successive iterates.

\begin{tabular}{ccc} 
Stage & $n$ & $\left|v_{n+1}-v_{n}\right|_{\max }$ \\
\hline 1 & 1 & .158628 \\
1 & 2 & .129673 \\
1 & 3 & \\
\hline 2 & 4 & $.277041 \times 10^{-2}$ \\
2 & 5 & $.346948 \times 10^{-3}$ \\
2 & 6 & \\
\hline 3 & 7 & $.328258 \times 10^{-5}$ \\
3 & 8 & $.799608 \times 10^{-7}$ \\
3 & 9 & \\
\hline
\end{tabular}

\section{$5 \quad$ Results and discussion}

The iterative scheme described in Section 4 was employed to construct the film radius profile $r(z)$ and temperature profile $s(z)$. Nine iterates were required to achieve a successive iterate tolerance of $10^{-6}$. Table 1 gives the maximum values of the successive iterates.

Figure 1 compares the isothermal approximate solution given in Section 3 with the thermal radial solution profile generated by the process of Section 4 building on the isothermal case.

Figure 2 shows how the radial solution profile is affected by changing the extrusion temperature (temperature at the die exit) of the polymer: as the temperature increases, the blow-up ratio increases.

Figure 3 shows how the temperature profile is affected by varying the extrusion temperature. The profiles decrease more rapidly as the extrusion temperature increases. 


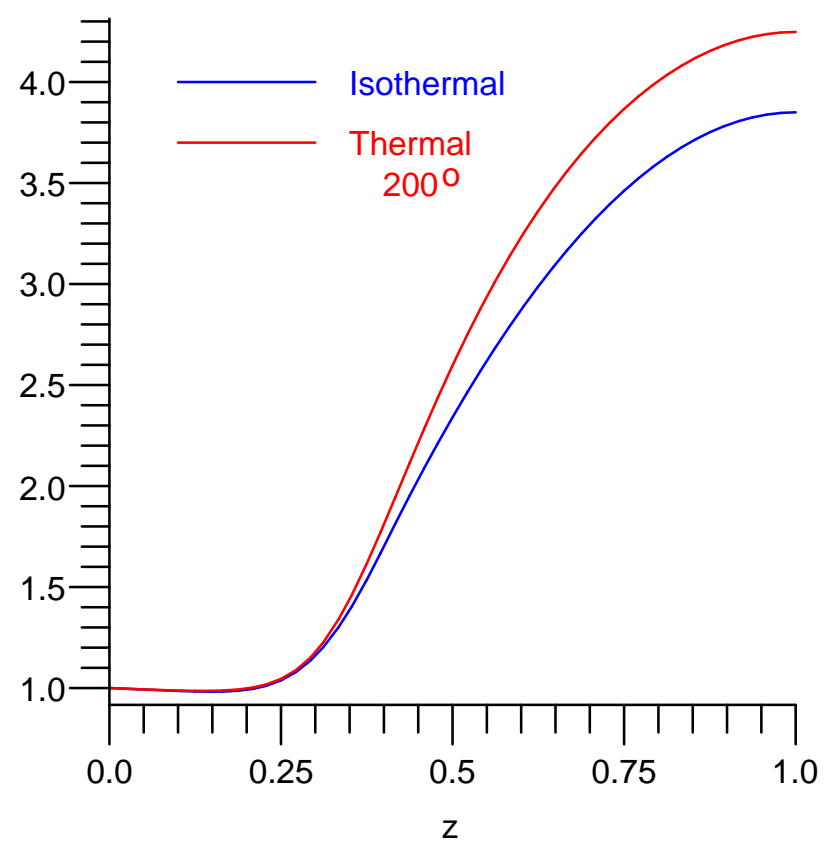

Figure 1: Comparison between isothermal perturbation approximation and thermal solution. 


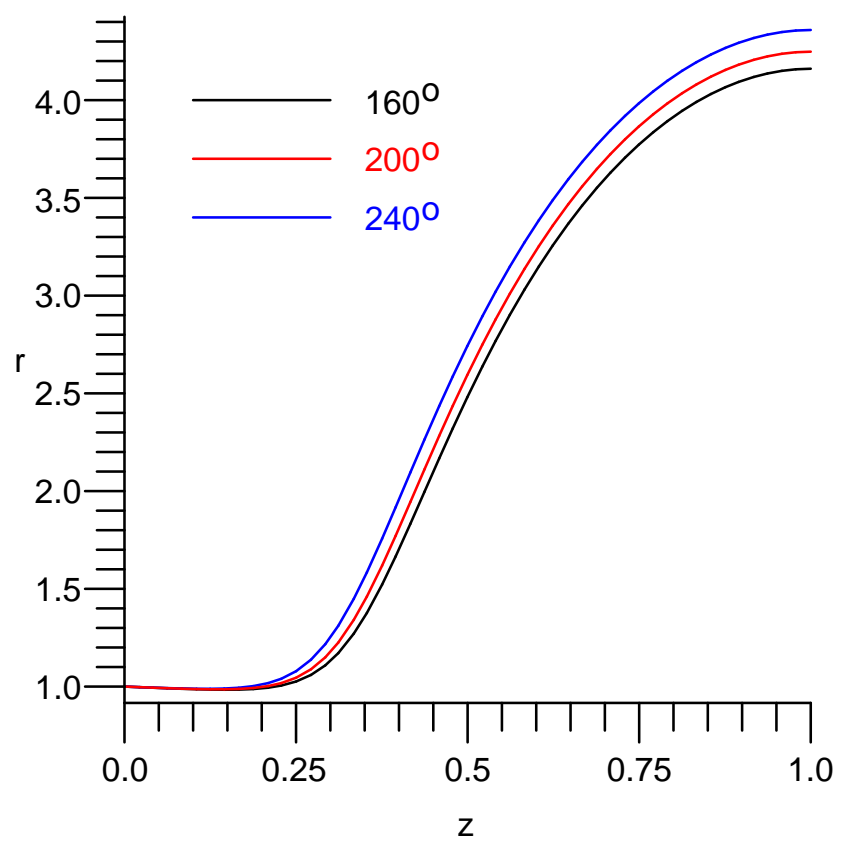

FiguRE 2: Thermal bubble radius profiles for varying temperatures. 


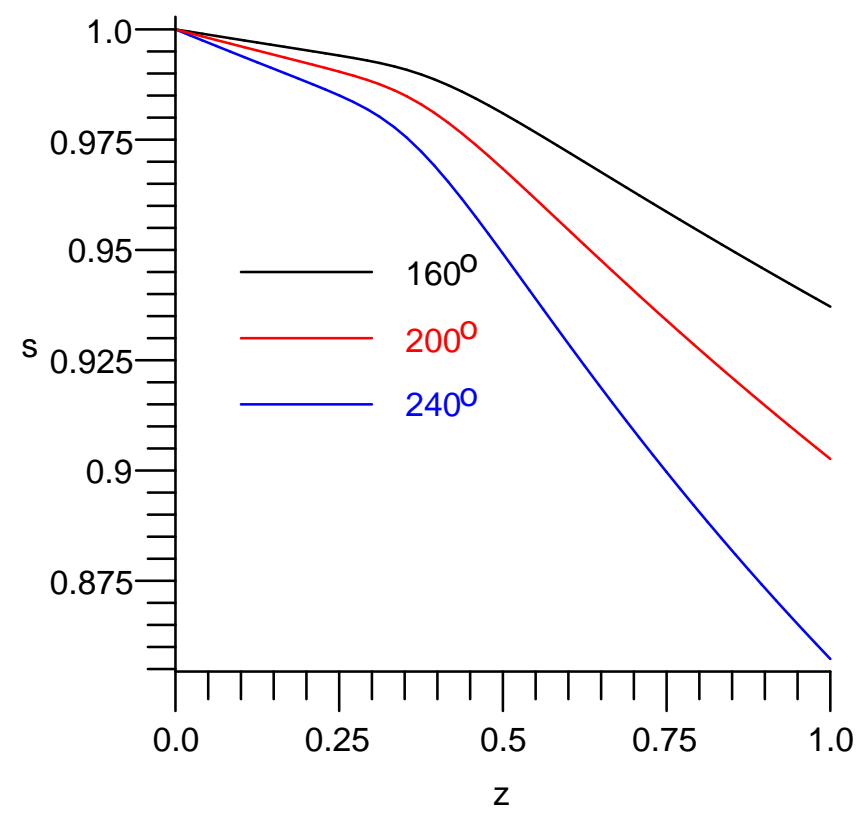

FiguRE 3: Thermal bubble temperature profiles for varying temperatures. 
The purpose of this article was to improve computation time to enable a more tractable solution mechanism. Use of the the Galerkin finite element method as above achieved this to a large degree, in that computation time was improved by a factor of approximately ten, when compared with previous calculations employing Maple's internal boundary value problem [3] to solve Equation (17). There, the complexity of the differential equation (17), due to the nonlinear $Q\left(R, v, v^{\prime}, v^{\prime \prime}\right)$ terms, along with having to solve the full equation each time, contributed to produce much longer computation times. In the present calculation, the employment of a finite element procedure reduces this complexity, firstly because the element matrix only has to be evaluated at each stage, and secondly, because the numerical integrations performed and resulting nodal values are found by solving a system of linear equations. This does not require differential operator manipulation. In either case, a highly nonlinear problem has been solved on a standard personal computer.

\section{References}

[1] Ervin V. J., Non-uniqueness of the Newtonian film blowing model, in preparation. C839

[2] Bennett J. C., Interior Layer Structure in the Blown Newtonian Film, Honours Thesis, School of Mathematical and Geospatial Science, RMIT, 2004. C839

[3] Bennett J. C. and Shepherd J. J., Analysis of the non-isothermal Newtonian model in the blown film process, ANZIAM Journal (E), 47, pp. C325-C338, 2006.

http://anziamj . austms.org.au/V47EMAC2005/Bennett C839, C840, C841, C842, C849 
[4] Luo X.-L. and Tanner R. I., A computer study of film blowing, Polymer Engineering and Science Vol. 25, No. 10, 1985, pp. 620-629. C839

[5] Muke S., Connell H., Sbarski I. and Bhattacharya S. N., Numerical modelling and experimental verification of blown film processing, Journal of Non-Newtonian Fluid Mechanics Vol. 116, 2003, pp. 113-138. doi:10.1016/j.jnnfm.2003.09.002 C838

[6] Pearson J. R. A., Mechanics of Polymer Processing, Elsevier Applied Science Publishers, 1985. C838

[7] Pearson J. R. A. and Petrie C. J. S., The flow of a tubular film. Part 1. Formal mathematical representation, Journal of Fluid Mechanics Vol. 40, 1970, pp. 1-19. C838

[8] Pearson J. R. A. and Petrie C. J. S., The flow of a tubular film. Part 2. Interpretation of the model and discussion of solutions, Journal of Fluid Mechanics Vol. 42, 1970, pp. 609-625. C838

[9] Shepherd J. J. and Bennett J. C., Interior layer structure in the Newtonian blown film, ANZIAM Journal 46(E), 2005, pp. C839-C853. http://anziamj . austms.org.au/V46/CTAC2004/Shep/ C839, C840, C841

[10] Shepherd J. J., Connell H. J. and Tam D. C. H., An interior layer in a film-blowing problem, Proceedings of the Fifth Biennial Engineering Mathematics and Applications Conference (EMAC2002), M. Pemberton, I. Turner, P. Jacobs (eds), The Institution of Engineers Australia, Brisbane, Queensland (2002), pp. 181-186. C839

[11] Tam D. C. H., Shepherd J. J. and Connell H. J., Modelling the film blowing process, Proceedings of the Fourth Biennial Engineering Mathematics and Applications Conference (EMAC2000), R L. May, G. F. Fitz-Gerald, I. H. Grundy (eds), The Institution of Engineers Australia, Melbourne (2000), pp. 271-274. C839 


\section{Author addresses}

1. J. C. Bennett, School of Mathematical and Geospatial Sciences, Royal Melbourne Institute of Technology, Melbourne, Australia. mailto:E58144@ems.rmit.edu.au

2. J. J. Shepherd, School of Mathematical and Geospatial Sciences, Royal Melbourne Institute of Technology, Melbourne, Australia. mailto: jshep@rmit.edu.au

3. W. F. Blyth, School of Mathematical and Geospatial Sciences, Royal Melbourne Institute of Technology, Melbourne, Australia. mailto:bill.blyth@rmit.edu.au 